\title{
IDENTIFICAÇÃO E MAPEAMENTO DE INSTITUIÇÕES DE ENSINO SUPERIOR E/OU PESQUISA AGRÍCOLA NO ESTADO DE SÃO PAULO ${ }^{1}$
}

\author{
Ricardo Firetti. APTA-SAASP, Polo Tecnológico Alta Sorocabana, Presidente Prudente-SP.
}

Eduardo Cardoso de Oliveira. APTA-SAASP, Polo Tecnológico Alta Sorocabana, Presidente PrudenteSP.

Maria Beatriz Machado Bonacelli. UNICAMP, Departamento de Política Científica e Tecnológica/Instituto de Geociências, Campinas-SP.

\section{RESUMO}

Tendo em vista conceitos referentes ao transbordamento de conhecimento ou spillover tecnológico presentes em aglomerações de empresas ou da produção, originadas em instituições de CT\&I e potencialmente apropriáveis pelo setor produtivo, ampliando sua capacidade competitiva, realizou-se estudo visando identificar e mapear unidades pertencentes a instituições de ensino superior e/ou pesquisa agrícola (públicas e particulares) localizadas em diferentes regiões do Estado de São Paulo, objetivando subsidiar informações a respeito da infraestrutura ligada a Ciência, Tecnologia e Inovação agrícola. Para tanto, utilizou-se como critério de territorialidade e regionalização, as Regiões de Governo do Estado de São Paulo, sendo que as instituições foram categorizadas em 04 em classes: Faculdades de Tecnologia; Unidades de Pesquisa; Faculdades Públicas; e Faculdades Particulares. Utilizou-se técnica de estatística descritiva nos dados categorizados e produziu-se mapa temático de localização. Foram identificadas 154 unidades vinculadas a instituições de ensino e/ou pesquisa relacionadas às Ciências Agrárias no Estado de São Paulo, e deste total quase 50\% dizem respeito a Unidades de Pesquisa (ligadas a instituições de pesquisa agrícola) e 30\% Faculdades Públicas. Observou-se grande concentração de unidades de ensino e/ou pesquisa em apenas 02 Regiões (Campinas e São Paulo), e que $48 \%$ das unidades identificadas encontram-se em apenas 08 Regiões, incluindo Presidente Prudente e Itapeva.

Palavras Chave: aglomeração; desconcentração administrativa; inovação agrícola; fontes de inovação

\begin{abstract}
This article relates around concepts about knowledge and technological spillover, present in clusters of firms or production, originated in RD institutions and potentially appropriated by the productive sector, increasing its competitiveness. There, the work objective to identify and to map units of the institutions higher education and/or agricultural research (public and private) located in different regions of Sao Paulo, aiming to subsidize information about the infrastructure linked to science, technology and agricultural innovation. Therefore, it was used as a criterion of territoriality and regionalization, the regions of Sao Paulo State Government. The institutions were categorized in 04 classes: Colleges of Technology; Research Units; Public Colleges; and Private Colleges. We used descriptive statistics technique in categorized data and was produced themed location map. There identified 154 units linked to educational institutions and/or research related to Agricultural Sciences in Sao Paulo, and of this total nearly 50\% relate to research units (linked to agricultural research institutions) and 30\% Public Colleges. There was a large concentration of in only 02 regions (Campinas and São Paulo), and that $48 \%$ of the identified units are in only 08 regions, including Presidente Prudente and Itapeva.
\end{abstract}

Keywords: agglomeration; administrative concentration; agricultural innovation; innovation

ÁREA 5: Mudanças técnicas, organizações e instituições

Classificação JEL: Q16 - R\&D; Agricultural Technology; Biofuels; Agricultural Extension Services

\footnotetext{
${ }^{1}$ Este trabalho integra pesquisa financiada pela FAPESP (Processo 2015/14310-7) intitulada "Embriões de sistemas locais de produção agrícola: mapeamento, caracterização e a interação entre as fontes de inovação”.
} 


\section{PROBLEMATIZAÇÃo}

Em sua análise sobre os "distritos industriais britânicos”, A. Marshall demonstrou que as firmas aglomeradas são capazes de se apropriarem de externalidades positivas (economias externas) geradas pela concentração de produtores, incrementando sua capacidade competitiva, algo que não se verificaria se estivessem atuando isoladamente. No entanto, segundo Garcia (2006), para Marshall, dentre as causas originais para a aglomeração (concentração) de empresas está a existência de condições naturais, tais como a disponibilidade de matéria-prima e de fontes de energia, ou facilidades no transporte, além da existência prévia de demanda na região.

De acordo com Araújo (2014) as externalidades positivas podem ser definidas como "custos ou benefícios decorrentes de uma atividade que afeta terceiros, mas que não optaram por incorrê-los”, ou seja, podem se originar de esforços de algumas empresas (ou organizações) mas serem apropriadas por diversas outras empresas locais. Desta forma, haveria três tipos de externalidades, decorrentes de: a) acesso a um maior número de fornecedores especializados; b) existência de denso conjunto de mão de obra qualificada; e c) maior disseminação de conhecimentos relacionados à atividade produtiva que levaria a processos de aprendizado e inovação mais eficientes, também chamados de transbordamentos, ou spillovers de conhecimento.

Ruffoni e Suzigan (2012), no entanto, alertam que o "local” pode ser fonte de aspectos negativos para a geração de inovações, e que parte das firmas ali presentes aproveitam de formas diferentes as supostas vantagens existentes nesse ambiente. Apontam críticas na literatura sobre a superestimação dos benefícios das economias externas a partir de dois argumentos centrais: a) externalidades podem impactar negativamente ou de formas diferentes as firmas de um mesmo segmento produtivo, dependendo da capacidade da própria firma em absorvê-las; e b) firmas geograficamente próximas podem beneficiar-se tanto de externalidades presentes no local, quanto de elementos externos ao local para a geração e/ou adoção de inovações.

O mesmo autor e colaboradores (GARCIA et al., 2011) entendem que a proximidade geográfica representa um estímulo relevante na geração e difusão de novos conhecimentos e capacitações entre os agentes econômicos, principalmente quando se verifica a presença concentrada de instituições de CT\&I, tendo em vista a possibilidade da oferta de um conjunto de insumos inovativos que se somam aos esforços de desenvolvimento tecnológico interno das empresas. Segundo Audretsch (1998), instituições de ensino e pesquisa (universidades, escolas tecnológicas e centros de P\&D) são responsáveis por parte dos transbordamentos de conhecimento, que tendem a ser restritos ao espaço geográfico e, com isso, podem ser considerados uma fonte de vantagem comparativa.

O pano de fundo desta suposição é que existe uma relação positiva entre a existência de recursos relativamente imóveis - como conhecimento, habilidades, estruturas institucionais e organizacionais -, e variações no crescimento e na performance econômica (BRESCHI e MALERBA, 2001).

Colocado de outra forma, pode-se dizer que existe uma relação entre geografia e inovação, ou seja, uma convergência entre a estrutura produtiva e a presença de organizações de CT\&I. (SUZIGAN, et al 2004; SUZIGAN et al., 2005; GARCIA et al.,2011). Nesse sentido, também é identificado que existe uma relação entre políticas de desenvolvimento regional e a formação de bases locais de CT\&I. Vieira (2010) com base em evidências empíricas coloca que uma política de desenvolvimento pode usar a CT\&I como ferramenta para gerar, agregar valor ao setor produtivo e ampliar a apropriação do valor produzido na região.

Segundo Salles-Filho et al. (2011), o setor agrícola é caracterizado por uma maior integração entre a geração e adoção de conhecimento, pois a pesquisa agrícola historicamente esteve articulada com o setor produtivo, mesmo que isto tenha ocorrido por intermédio de instrumentos e instituições de assistência técnica e extensão rural. Seu conjunto, incluindo setores a montante (insumos) e a jusante (beneficiamento, industrialização e distribuição) poderia ser visto como um Sistema Setorial de Inovação ${ }^{2}$.

\footnotetext{
${ }^{2}$ O conceito de sistema setorial de inovações permite a utilização de uma visão multidimensional integrada e dinâmica da inovação. Sistemas setoriais apresentam três dimensões que afetam tanto a geração e adoção de novas tecnologias quanto a
} 
Para os autores, a unidade de produção agrícola (ou estabelecimento agropecuário), embora não seja um local dinâmico de criação de novo conhecimento, pode ser considerada como fonte de inovação na agricultura por ser o "locus no qual todo conjunto de tecnologias agrícolas se reúne e se consubstancia, com uma base técnica coerente de produção". Ponderam ainda que "o learning by using e o leraning by doing são as principais formas de influência dessa fonte na base técnica de produção na agricultura”.

Entretanto, as fontes institucionais públicas ${ }^{3}$, normalmente são responsáveis pela geração e difusão de tecnologia genética e de práticas agrícolas (técnicas de cultivo e criação), além de parte importante da pesquisa básica voltada ao conhecimento novo para o setor agrícola. Salles-Filho et al. (2011) consideram que em função da forma e conteúdo aplicado desse conhecimento (e tecnologias), a pesquisa agrícola poderia ser exemplo de sucesso na articulação entre governo, pesquisa e produção.

Notadamente, o Estado de São Paulo abriga uma rede de instituições acadêmicas e de pesquisa de referência internacional e um parque informacional formado por setores industriais e de serviços intensivos. Resultado de investimentos estaduais e federais sistemáticos, ao longo de décadas, esse Sistema demonstra sua excelência no fato de ser responsável por grande parte da produção científica brasileira (QUADROS et al., 2000).

Por sua vez, essa infraestrutura, também denominada na literatura por Sistema Paulista de Ciência, Tecnologia e Inovação Agrícola (SALLES et al., 2011), embora inexista formalmente, é formada por um conjunto de instituições públicas (APTA, UNESP, UNICAMP, USP, FATECs, ETECs, UFSCar, Embrapa, IFCT ${ }^{4}$, Unifesp, e UFABC) e particulares (FGV, UNOESTE, UNIFRAN, UNISA, UNIP, UNICASTELO, FUNDECITRUS, CTC-CANA), com atuação em ensino e/ou pesquisa, que estão distribuídas pelo território estadual atuando em diferentes áreas das Ciências Agrárias, Medicina Veterinária, Zootecnia e Recursos Pesqueiros, Administração e Gestão Rural, Economia Agrícola, Ciência e Engenharia de Alimentos. Segundo os autores, "o SPInA é bastante complexo e denso, pois trata-se de um sistema com formação histórica ampla (mais de 120 anos), com organizações públicas de importância crucial não apenas para o cenário do desenvolvimento agrícola de São Paulo, mas também para o país”.

O estado possui ainda a principal Organização Estadual de Pesquisa Agropecuária pertencente ao Sistema Nacional de Pesquisa Agropecuária (SNPA) ${ }^{5}$ : a APTA - Agência Paulista de Tecnologia dos Agronegócios (SICSÚ; SILVEIRA, 2013).

A APTA é órgão da Administração Direta do Governo do Estado de São Paulo e vinculada à Secretaria de Agricultura e Abastecimento (SAA). Parte de sua missão está direcionada sinteticamente a "gerar, adaptar e transferir conhecimentos científicos e tecnológicos para sustentação e ampliação da competitividade das cadeias de produção dos agronegócios paulistas, com ênfase no agronegócio familiar” (SÃO PAULO, 2002). Reúne 47 centros de P\&D com atribuições no desenvolvimento de trabalhos científicos e tecnológicos, e 2.440 servidores dos quais 780 pesquisadores.

Sua origem está atrelada à Coordenadoria de Pesquisa Agropecuária da SAA, aproveitando a estrutura remanescente de institutos de pesquisa do Estado de São Paulo: Instituto Agronômico de Campinas, Instituto Biológico, Instituto de Zootecnia, Instituto de Economia Agrícola, Instituto de Pesca e Instituto de Tecnologia de Alimentos. Atualmente, a APTA é composta pela unidade de coordenação (Gabinete do Coordenador), unidade de planejamento e avaliação (Departamento de Gestão Estratégica), unidades de $P \& D$ de abrangência estadual (Institutos de Pesquisa), e unidades de P\&D de abrangência regional (Polos Regionais de Desenvolvimento Tecnológico dos Agronegócios).

Para Gonçalves (2006, p.80) as unidades de pesquisa de abrangência regional poderiam “impulsionar as economias regionais, estruturando consistente rede desconcentrada de inovação, focando

organização da inovação e produção nos seguintes níveis setoriais: conhecimento, atores e redes e instituições (MALERBA, 2003).

3 "Incluem as universidades, instituições de pesquisa (como as organizações nacionais ou estaduais de pesquisa agrícola), órgãos de assistência técnica e extensão rural (Ater)” (SALLES-FILHO et al., 2011).

${ }^{4}$ Instituto Federal de Ciência e Tecnologia.

${ }^{5}$ O SNPA é formado pela Empresa Brasileira de Pesquisa Agropecuária (que coordena o sistema) e pelas Organizações Estaduais de Pesquisa Agropecuária (OEPAs), universidades e institutos de pesquisa de âmbito federal e estadual e ainda por outras organizações, públicas e privadas, direta ou indiretamente vinculadas à pesquisa agropecuária (VIEIRA-FILHO, 2012). 
as cadeias de produção da agricultura regional e a criação de novos agronegócios”. Todavia, o autor pondera que o tratamento dado à regionalidade nas ações do Governo do Estado de São Paulo mostravam problemas de convergência e foco, tendo em vista que "enorme fragmentação de ações locais dos vários órgãos, não por serem vinculados a secretarias e instituições diferentes, mas por não haver qualquer nexo compatibilizador dos diversos espaços geográficos, levando cada qual a planejar e a agir como se os demais órgãos não existissem”.

\section{OBJETIVOS}

Este trabalho objetiva identificar e mapear unidades pertencentes a instituições de ensino superior e/ou pesquisa agrícola (públicas e particulares) localizadas nas Regiões de Governo do Estado de São Paulo, visando subsidiar informações a respeito da infraestrutura ligada a Ciência, Tecnologia e Inovação agrícola.

\section{METODOLOGIA}

Este trabalho é resultado parcial de pesquisa exploratória sobre instituições públicas e privadas que atuam junto ao ensino superior e pesquisa em áreas relacionadas às Ciências Agrárias. Segundo Gil (2009), este tipo de estudo "têm a finalidade de desenvolver, esclarecer e modificar conceitos e ideias, tendo em vista a formulação de problemas mais precisos ou hipóteses pesquisáveis para estudos posteriores”.

Foram identificadas instituições de ensino superior e pesquisa (públicas e privadas) e instituições de pesquisa (públicas), independentemente de sua vinculação com as esferas da federação (municipal, estadual ou federal). Segundo Audretsch (1998), estas instituições são responsáveis direta ou indiretamente por parte dos transbordamentos de conhecimento no espaço geográfico.

O levantamento foi realizado em armazéns de dados oficiais dentre as quais estão os microdados disponibilizados pelo Ministério da Educação (MEC), Coordenação de Aperfeiçoamento de Pessoal de Nível Superior (Capes), Empresa Brasileira de Pesquisa Agropecuária (EMBRAPA), Instituto Nacional de Estudos e Pesquisas Educacionais Anísio Teixeira (INEP), Conselho Nacional de Desenvolvimento Científico e Tecnológico (CNPq), além de outros sítios de internet de instituições e consultas documentais. Para tanto, considerou-se o ano base de 2014.

Com base informações advindas da experiência do pesquisador principal, tendo em vista sua atuação profissional no âmbito de CT\&I e prevista metodologicamente por Gil (2009), para identificar as estruturas de ensino superior tecnológico, utilizou-se de informações do sitio de internet do Centro Paula Souza, notadamente o "Perfil das Faculdades de Tecnologia (FATECs) e "Onde Estudar", ambos disponibilizados pelo Governo do estado de São Paulo, mais especificamente pela Secretaria de Desenvolvimento, Ciência, Tecnologia e Inovação. Utilizou-se ainda, o sitio do Instituto Federal de Educação, Ciência e Tecnologia de São Paulo - IFSP, notadamente a página de “Cursos Tecnológicos”, observando-se aqueles os cursos que têm aderência aos segmentos de insumos agropecuários, produção agropecuária e transformação do produto agropecuário.

As instituições públicas de pesquisa em ciências agrárias, denominadas neste levantamento como "Unidades de Pesquisa”, compreendem as unidades da Empresa Brasileira de Pesquisa Agropecuária (EMBRAPA) e as "unidades básicas de ciência e tecnologia”6 da Agência Paulista de Tecnologia dos Agronegócios (APTA). Para as unidades da EMBRAPA, os dados foram coletados no site oficial da instituição, enquanto que para as unidades relacionadas à APTA, utilizou-se o Decreto $\mathrm{N}^{0} 46.488 / 2002$ (SÃO PAULO, 2002) que "reorganiza a Agência Paulista de Tecnologia dos Agronegócios (APTA), da Secretaria de Agricultura e Abastecimento, e dá providências correlatas”.

Em relação às Universidades Privadas, levou-se em consideração apenas as Universidades com algum programa de pós-graduação reconhecido pela CAPES, com concessão de bolsas, sendo que os dados foram extraídos pelo sistema de informações georreferenciadas (GEOCAPES).

\footnotetext{
${ }^{6}$ De acordo com o Decreto 46.488/2002 que reorganizou a APTA no âmbito da administração direta do Governo do Estado, são consideradas como Unidades Básicas de Ciência e Tecnologia: Centros de Pesquisa e Desenvolvimento; Centros de Análise e Pesquisa Tecnológica do Agronegócio; Centros Avançados de Pesquisa Tecnológica do Agronegócio; Polos Regionais de Desenvolvimento Tecnológico dos Agronegócios; e Unidades de Pesquisa e Desenvolvimento.
} 
Utilizou-se como critério de territorialidade e regionalização, as Regiões de Governo do Estado de São Paulo (RG) identificadas de acordo com a organização oferecida no sitio de internet da Fundação Sistema Estadual de Análise de Dados (SEADE, 2016). Consistem em microrregiões geográficas que compõem as Regiões Administrativas (RA) do estado. A escolha desse critério foi baseada pelo fato de serem oficialmente utilizadas pelas Secretarias de Estado, em especial, a de Planejamento e Gestão.

Embora a Região Metropolitana de São Paulo não seja oficialmente considerada como Região de Governo, para efeito deste estudo foi incluída como tal.

Os dados obtidos no levantamento foram organizados em forma de tabelas em software para elaboração de planilhas eletrônicas, visando facilitar a utilização em outros aplicativos e sistemas. Para efeito ilustrativo e de utilização no mapeamento, as instituições foram categorizadas em 04 em classes: Faculdades de Tecnologia (instituições de ensino superior tecnológico); Unidade de Pesquisa (instituições públicas de pesquisa); Faculdades Públicas (faculdades de ensino e pesquisa vinculadas às Universidades Públicas); e Faculdades Particulares (faculdades de ensino e pesquisa particulares).

Foram utilizadas técnicas de estatística descritiva nos dados categorizados, sendo obtidos valores médios de unidades por Região de Governo e elaborados histogramas de frequência, visando observar a distribuição e concentração das unidades de ensino e/ou pesquisa.

O mapa temático de localização das unidades identificadas foi produzido no Sistema de Informações Geográficas TerraView - Política Social, versão 4.2.2, disponibilizado pelo Centro de Estudos da Metrópole (CEM, 2015). Trata-se de uma ferramenta voltada para a produção de análises a partir de dados demográficos, econômicos e sociais, no qual a geração de mapas e gráficos é feita a partir de bases cartográficas de domínio público.

\section{RESULTADOS OBTIDOS}

Foram identificadas 154 unidades vinculadas a instituições de ensino e/ou pesquisa relacionadas às Ciências Agrárias no Estado de São Paulo. Deste total, quase 50\% (75) dizem respeito a Unidades de Pesquisa (ligadas a instituições de pesquisa agrícola); 30\% Faculdades Públicas; 15\% relacionadas às Faculdades de Tecnologia e apenas 7\% de Faculdades Particulares. Em média, observou-se a existência de 3,58 unidades/Região de Governo.

No histograma de frequências (Figura 1) percebe-se grande concentração de unidades de ensino e/ou pesquisa em apenas 02 Regiões de Governo. Apenas 04 Regiões não possuem qualquer instituição representada; enquanto que a maior frequência incidente está vinculada à pelo menos 02 unidades em 11 Regiões de Governo. 


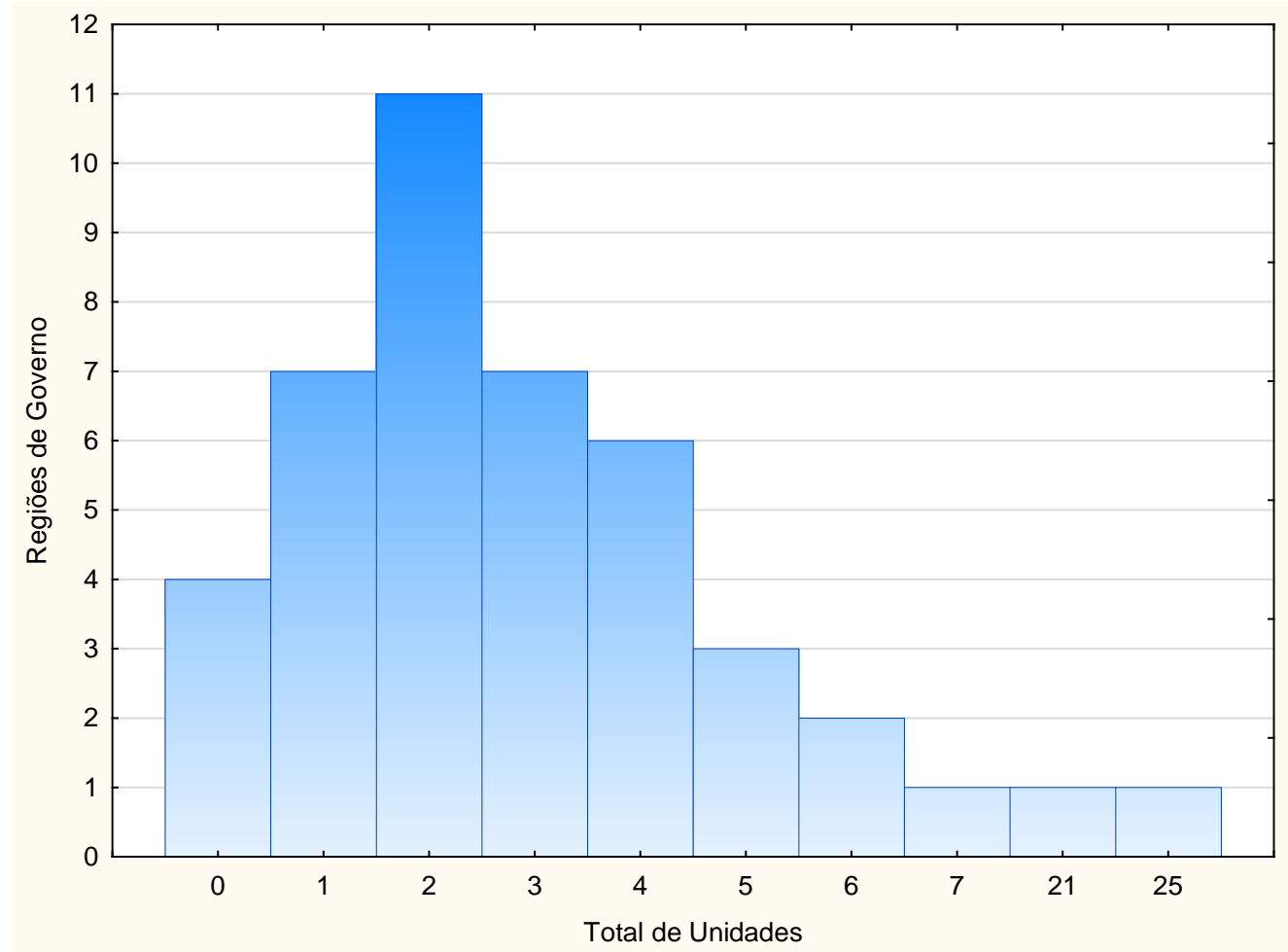

Figura 1. Histograma de frequência de observações do total de unidades de ensino superior e/ou pesquisa nas Regiões de Governo.

Fonte: Resultados da Pesquisa.

A concentração das unidades de ensino e/ou pesquisa mencionada ocorrem na Região de Governo de Campinas (25 unidades) e Metropolitana de São Paulo (21).

O resultado obtido para as Regiões de Campinas e São Paulo é semelhante ao encontrado no trabalho de Suzigan et al (2004), que a partir de indicadores quantitativos regionalizados mostraram a ocorrência de um padrão na distribuição regional das atividades de C,T\&I ao longo dos eixos das principais rodovias do estado, e no entorno de áreas metropolitanas, com ênfase em São Paulo e Campinas, muito embora esses autores estivessem pesquisando indicadores envolvendo áreas além das Ciências Agrárias.

Entretanto, é importante mencionar que essa concentração era mais acentuada há menos de três décadas atrás, antes da reorganização da pesquisa agrícola vinculada à Secretaria de Agricultura e Abastecimento do Estado de São Paulo, e da interiorização das estruturas universitárias, como as unidades diferenciadas da Universidade Estadual Paulista Júlio de Mesquita Filho (UNESP) e das Faculdades de Tecnologias (FATECs).

Ao observar-se apenas a categoria de Unidades de Pesquisa, formada por unidades ligadas às Organizações Públicas de Pesquisa (OPP), especificamente a Empresa Brasileira de Pesquisa Agropecuária (EMBRAPA) e a Agência Paulista de Tecnologia dos Agronegócios (APTA) é possível notar a existência de concentração ainda maior do que a anteriormente observada, pois apenas uma Região de Governo, neste caso novamente a de Campinas, agrega 22 Unidades de Pesquisa (Figura 2). Observa-se ainda que 13 Regiões de Governo não possuem Unidades de Pesquisa instaladas, mas, em contrapartida, ao menos 15 Regiões possuem uma unidade; 08 Regiões apresentam 02 unidades; e 05 Regiões têm 03 unidades.

Este fato já havia sido explorado, especificamente pensando na agricultura paulista, por Gonçalves (2005), ao afirmar que “a regionalização da pesquisa pública paulista para o agronegócio tem sua origem na necessidade de reduzir a concentração de instituições e centros de pesquisa no eixo Anhanguera Bandeirantes". Para o autor, a estrutura pública deveria aprofundar a desconcentração para "gerar e adaptar inovações com diferenciação de produtos e processos visando criar vantagens de origem, além de atuar na irradiação regional das inovações disponíveis, reduzindo disparidades entre as diferentes regiões do estado”. 


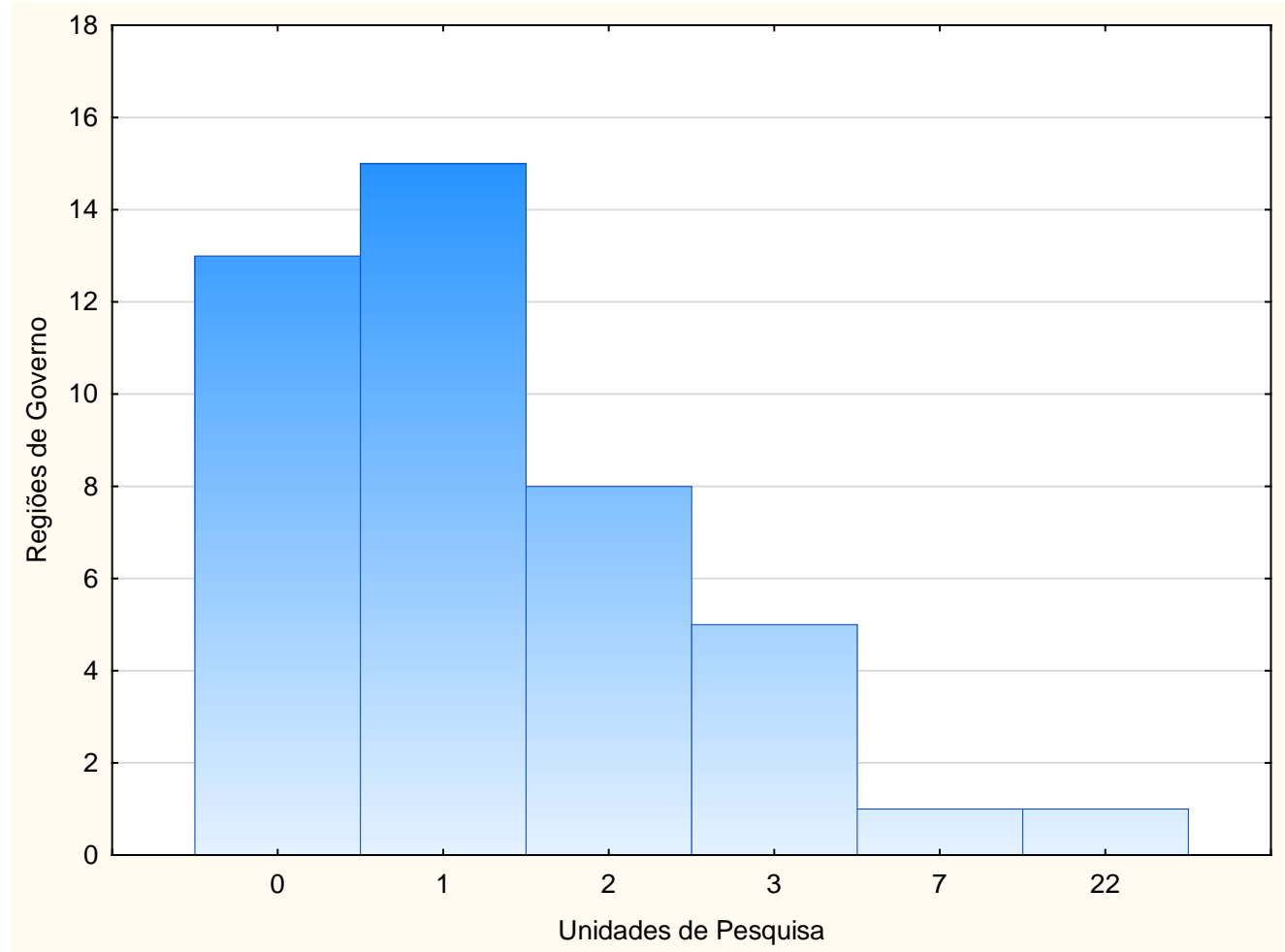

Figura 2. Histograma da frequência de observações do total de Unidades de Pesquisa nas Regiões de Governo.

Fonte: Resultados da Pesquisa.

Parte dessa concentração pode ser explicada pela natureza e trajetória das Organizações Públicas de Pesquisa que compõem esta categoria: Embrapa e APTA.

No caso da EMBRAPA, esta possui 06 unidades de pesquisa em território paulista, das quais 04 concentradas na Região de Governo de Campinas e outras 02 na de São Carlos, respectivamente: Embrapa Monitoramento por Satélite (criada em 1989), Informática Agropecuária (1993), Gestão Territorial (2011); e Embrapa Pecuária Sudeste (1975), e Instrumentação Agropecuária (1984).

Segundo Alves (2010), a Embrapa possui um modelo descentralizado na dimensão territorial, mas concentrado de organização das Unidades de Pesquisa, que são especializadas em produtos, recursos e temáticas. Para o autor essa forma organizacional "teve papel importante ao evitar a dispersão de esforços, que é um de seus fundamentos e é uma forma objetiva de sinalizar prioridades de pesquisa”.

Basicamente a criação de novas unidades da Embrapa ao longo do tempo em São Paulo aproveitou a infraestrutura original já existente, ou as redes de relacionamento pré-estabelecidas com outras instituições federais e estaduais, tais como a UNICAMP e o Instituo Renato Archer, ligado ao Ministério de Ciência, Tecnologia, Inovações e Comunicações.

No caso da APTA, a opção foi de transformar estações (fazendas) experimentais em novos centros regionais de pesquisa e desenvolvimento agrícola distribuídos atualmente em 14 regiões paulistas (Polos Regionais de Desenvolvimento Tecnológico). Os próprios Institutos de Pesquisa que compõem a agência desde o momento de sua criação, passaram por um processo de descentralização, iniciado em 1998 que culminaria com a criação de Centros de Pesquisa e Desenvolvimento, Centros de Análise e Pesquisa Tecnológica e Centros Avançados de Pesquisa Tecnológica em 2002.

As Faculdades Públicas formam a segunda categoria com maior número de unidades de instituições de ensino e/ou pesquisa, com 46 observações. Fizeram parte do levantamento, organizações das esferas municipal, estadual e federal. Ao contrário da categoria anterior, neste caso ocorre uma melhor distribuição das unidades (faculdades e institutos de ensino) nas Regiões de Governo (Figura 3), em decorrência da localização dos Campus da Universidade de São Paulo (USP) no interior do Estado, mas principalmente pela natureza descentralizada de atuação da Universidade Estadual Paulista (UNESP), que por si só representa 37\% da categoria (17 unidades). 
Destaca-se a participação de instituições da esfera municipal, algumas delas muito representativas regionalmente, fato que será destacado mais adiante em análise do mapeamento. Foram identificadas 06 unidades de ensino superior nessas condições, número semelhante à de unidades federais. Todavia, a maior parte dessas instituições está localizada em municípios pequenos, com populações variando entre 30.000 e 142.000 habitantes. Fato este que suscita outros questionamentos relacionados à capacidade, necessidade ou interesse de cidades médias do interior do Estado de São Paulo em oferecer ensino superior em áreas estratégicas não contempladas pela estrutura estadual e/ou federal.

Para Gonçalves (2006, p.80), no campo da formação de capital intelectual, as iniciativas de instalação das Unidades Diferenciadas da Universidade Estadual Paulista "Júlio de Mesquita Filho" (UNESP) e das Faculdades de Tecnologia (FATECs) do Centro Estadual de Educação Tecnológica "Paula Souza”, aprofundaram a interiorização da formação de nível superior com o oferecimento de cursos focados nas realidades locais.

Observou-se que 19 Regiões de Governo possuem ao menos uma unidade de ensino superior pública relacionada às Ciências Agrárias, sendo que outras 07 Regiões apresentam até duas unidades, nesse caso especialmente, geralmente com a presença de uma instituição municipal. Apenas a Região Metropolitana de São Paulo possui maior concentração de unidades desta natureza (07).

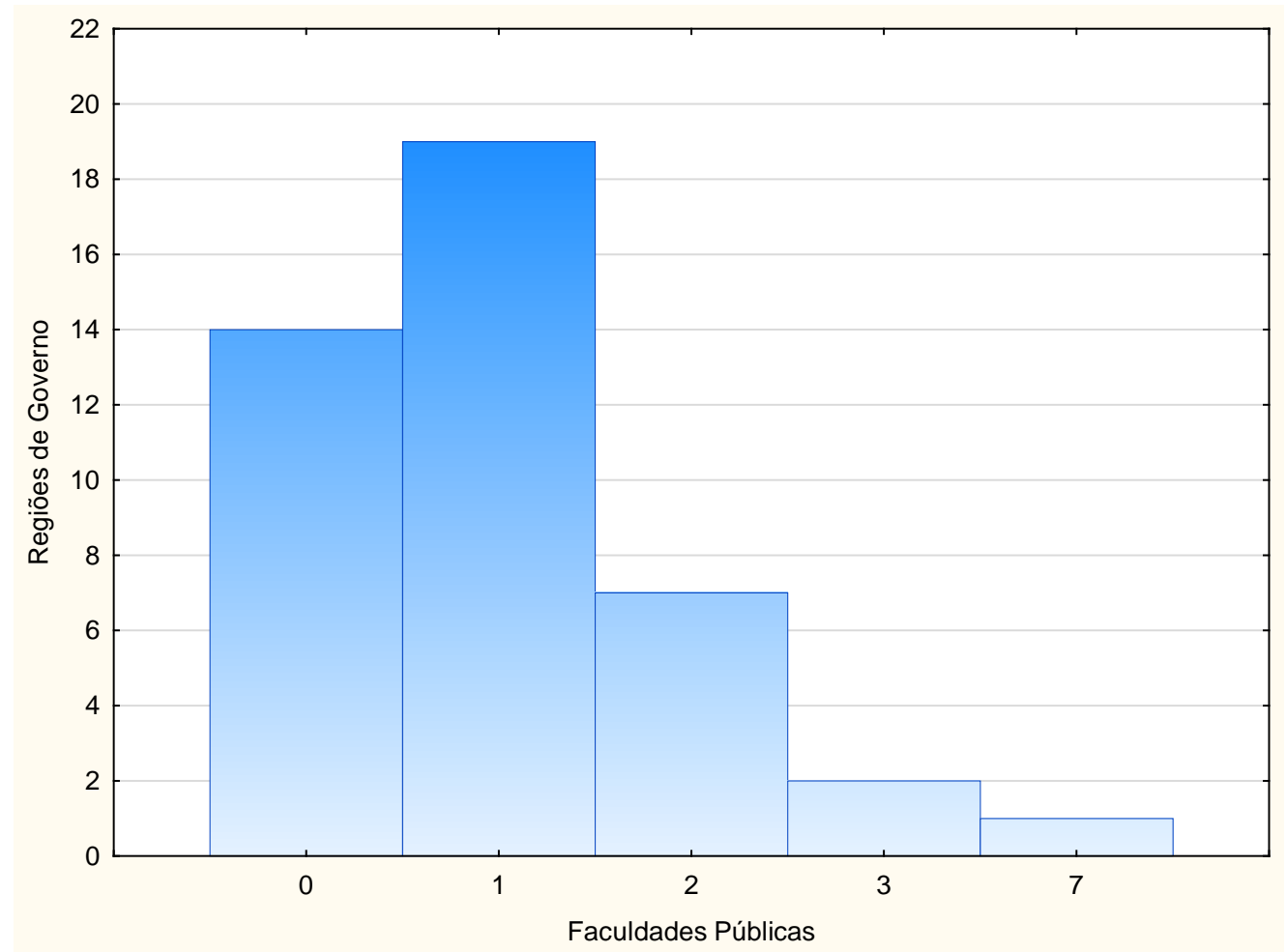

Figura 3. Histograma da frequência de observações do total de Faculdades Públicas nas Regiões de Governo.

Fonte: Resultados da pesquisa.

As Faculdade de Tecnologia formam a terceira categoria analisada e são compostas basicamente pelo Instituto Federal de Educação, Ciência e Tecnologia de São Paulo (IFSP), autarquia federal de ensino, e as Faculdades de Tecnologia do Centro Paula Souza (FATEC), autarquia do Governo do Estado de São Paulo vinculada à Secretaria de Desenvolvimento Econômico, Ciência, Tecnologia e Inovação (SDECTI).

Ocorre que das 22 unidades de ensino superior tecnológico identificadas, 86\% são da esfera estadual. Desta forma, a FATEC, ao lado da APTA, podem ser consideradas como as instituições matrizes com maior número de unidades relacionadas às Ciências Agrárias, embora qualitativamente ou a partir de análises quantitativas mais aprofundadas estes critérios possam perder valor.

Ao menos 16 Regiões de Governo têm instaladas unidades de Faculdades de Tecnologia, sendo esta categoria a de menor concentração geográfica. 


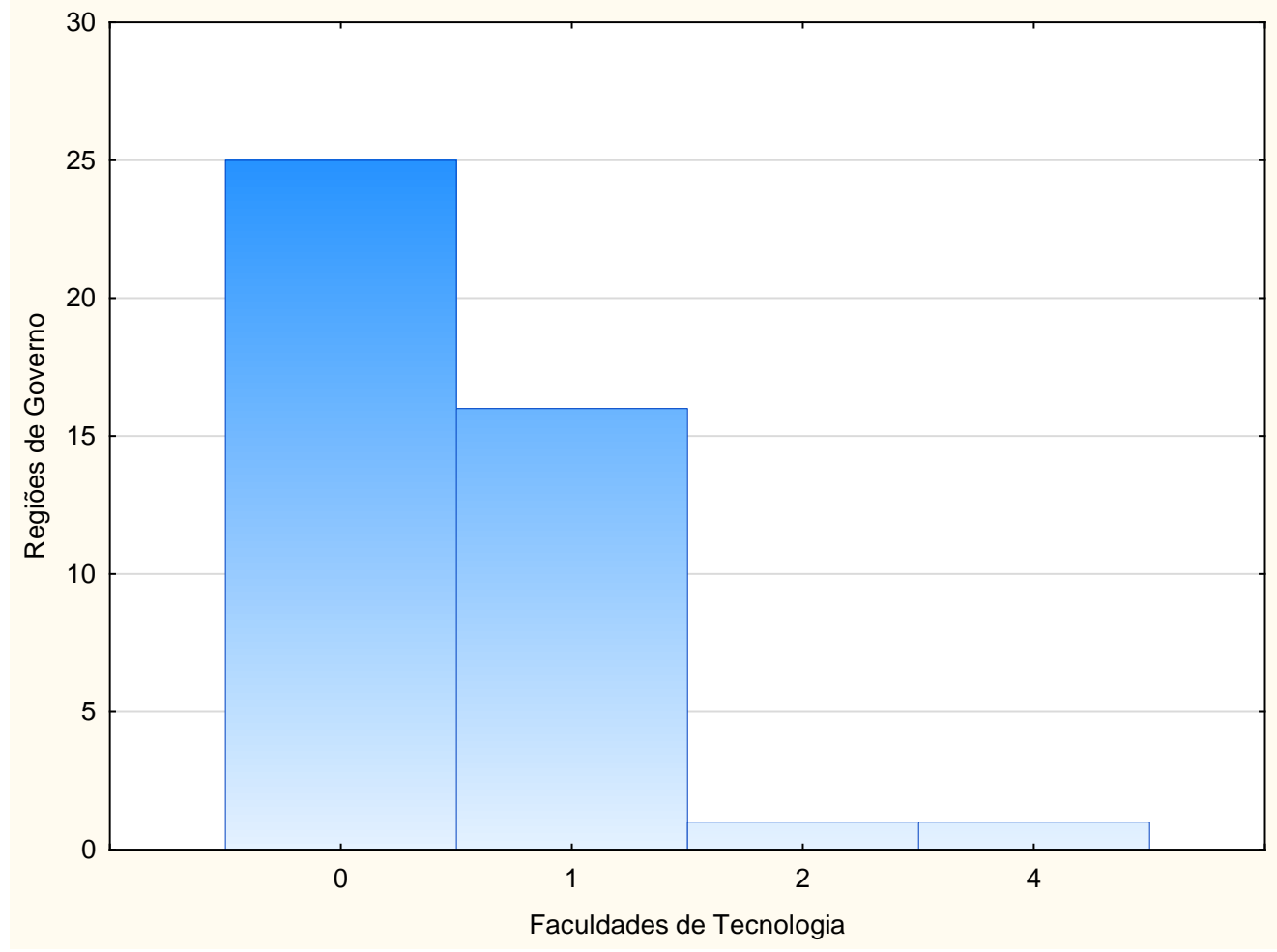

Figura 4. Histograma da frequência de observações do total de Faculdades de Tecnologia nas Regiões de Governo.

Fonte: Resultados da pesquisa.

No que se refere às Faculdades Particulares, foram identificadas 11 unidades no estado (7,1\% do total). Com exceção da Região Metropolitana de São Paulo, que concentra 03 unidades, outras 08 Regiões de Governo apresentam apenas uma instituição particular, evidenciando a desconcentração de instituições desta categoria.

Cabe ressaltar a importância que as instituições particulares possuem em âmbito regional, pois por vezes são atores locais de elevada representatividade e importância no desenvolvimento agrícola. Destaca-se por exemplo a Região Geográfica de Franca (Figura 05) que possui apenas uma instituição de ensino e/ou pesquisa em Ciências Agrárias (Universidade de Franca), com mais de 16 mil alunos distribuídos entre os cursos de graduação, tecnológicos, a distância, especializações, mestrado e doutorado, e abrigando mais de 500 docentes, cuja titulação ultrapassa $60 \%$ de mestres e doutores.

Observando o mapeamento gerado com a distribuição proporcional das unidades de ensino e/ou pesquisa em Ciências Agrárias nas Regiões de Governo do Estado de Paulo, pode-se verificar efetivamente a ocorrência de concentração de instituições em Campinas e São Paulo, assim como mencionado anteriormente, e corroborando com os trabalhos de Suzigan et al. (2004) e Gonçalves (2005).

De maneira geral o mapeamento reforça o pressuposto de que exista uma relação positiva entre a existência de recursos relativamente imóveis (conhecimento, habilidades, estruturas institucionais e organizacionais), e performance econômica (BRESCHI e MALERBA, 2001), em sinergia ao que afirmava Gonçalves (2006), de que os municípios mais desenvolvidos estão concentrados na linha que une os espaços metropolitanos da Capital e de Campinas e prolongam-se no sentido de Ribeirão Preto, e que o "rompimento com esse ciclo reprodutor de aprofundamento das disparidades regionais" exigiria o redesenho das instituições públicas a partir da interiorização das estruturas.

Percebe-se também que apenas a Região de Governo de Presidente Prudente e a Metropolitana de São Paulo possuem unidades das quatro categorias observadas, o que poderia representar oportunidade àquela região do extremo oeste do estado, tendo em vista suas características econômicas alicerçadas na produção agropecuária e serviços, e segundo Firetti et al. (2010) considerada uma das últimas fronteiras do desenvolvimento paulista pela Secretaria de Economia e Planejamento do Estado de São Paulo.

Garcia e colaboradores (2011) entendem que a proximidade geográfica representa um estímulo relevante na geração e difusão de novos conhecimentos e capacitações entre os agentes econômicos, 
principalmente quando se verifica a presença concentrada de instituições de CT\&I, tendo em vista a possibilidade da oferta de um conjunto de insumos inovativos que se somam aos esforços de desenvolvimento tecnológico interno das empresas.

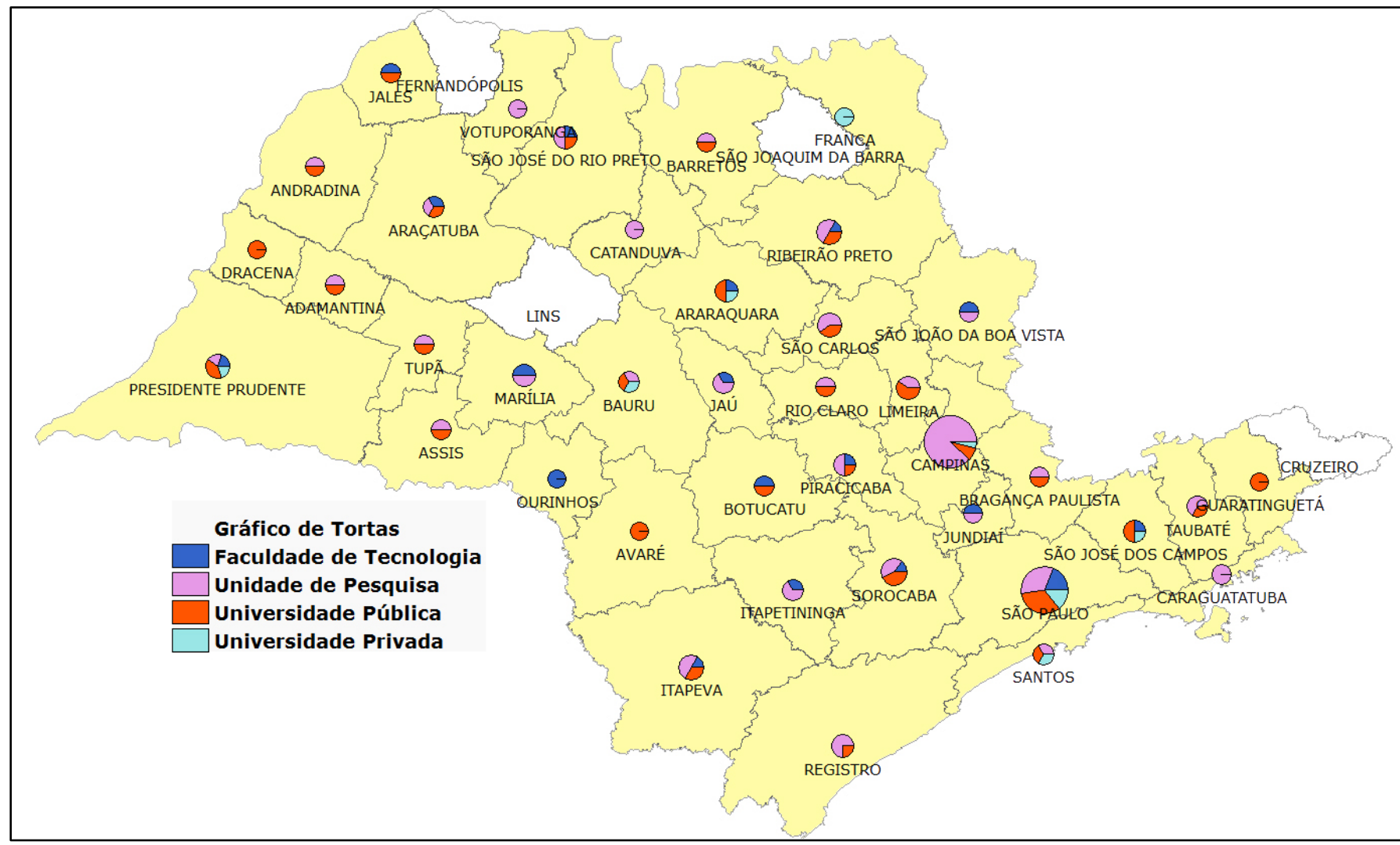

Figura 05. Distribuição proporcional de categorias de unidades de ensino superior e/ou pesquisa em Ciências Agrárias nas Regiões de Governo do Estado de São Paulo.

Com base nos resultados da Figura 5, e visando identificar quais Regiões de Governo concentram as unidades de ensino superior e/ou pesquisa em Ciências Agrárias, organizamos os dados obtidos em relação a frequência das unidades nas Regiões de governo, de maneira crescente, obtendo-se um diagrama no qual observa-se (Figura 6), inicialmente, que 48\% das unidades identificadas encontram-se em apenas 08 Regiões de Governo: Limeira, Presidente Prudente, São Carlos, Itapeva, Ribeirão Preto, Sorocaba, São Paulo e Campinas.

Desta forma, torna-se necessário destacar a presença das Regiões de Governo de Itapeva e Presidente Prudente nesse agrupamento, tendo em vista estarem localizadas geograficamente distantes dos "municípios mais desenvolvidos concentrados na linha que une os espaços metropolitanos da Capital e de Campinas e prolongam-se no sentido de Ribeirão Preto" (Gonçalves, 2006). Notadamente essas Regiões de Governo tem na produção agropecuária e nas agroindústrias suas principais fontes econômicas para geração de empregos e renda.

Mesmo sem elementos quantitativos e qualitativos suficientes que possam embasar a afirmação a seguir, tendo em vista a natureza exploratória desta pesquisa, pode-se entender que, direta ou indiretamente, o processo de desconcentração das instituições de ensino superior e/ou pesquisa ocorrido no Estado de São Paulo, a partir da reorganização da pesquisa agrícola e ampliação das unidades da UNESP e FATEC, criou condições mínimas para transbordamento de conhecimentos científicos e tecnológicos nas Regiões de Governo de Presidente Prudente e Itapeva.

Lembrando que, segundo Audretsch (1998), instituições de ensino e pesquisa (universidades, escolas tecnológicas e centros de $\mathrm{P} \& \mathrm{D})$ são responsáveis por parte dos transbordamentos de conhecimento, que tendem a ser restritos ao espaço geográfico e, com isso, podem ser considerados uma fonte de vantagem comparativa. 


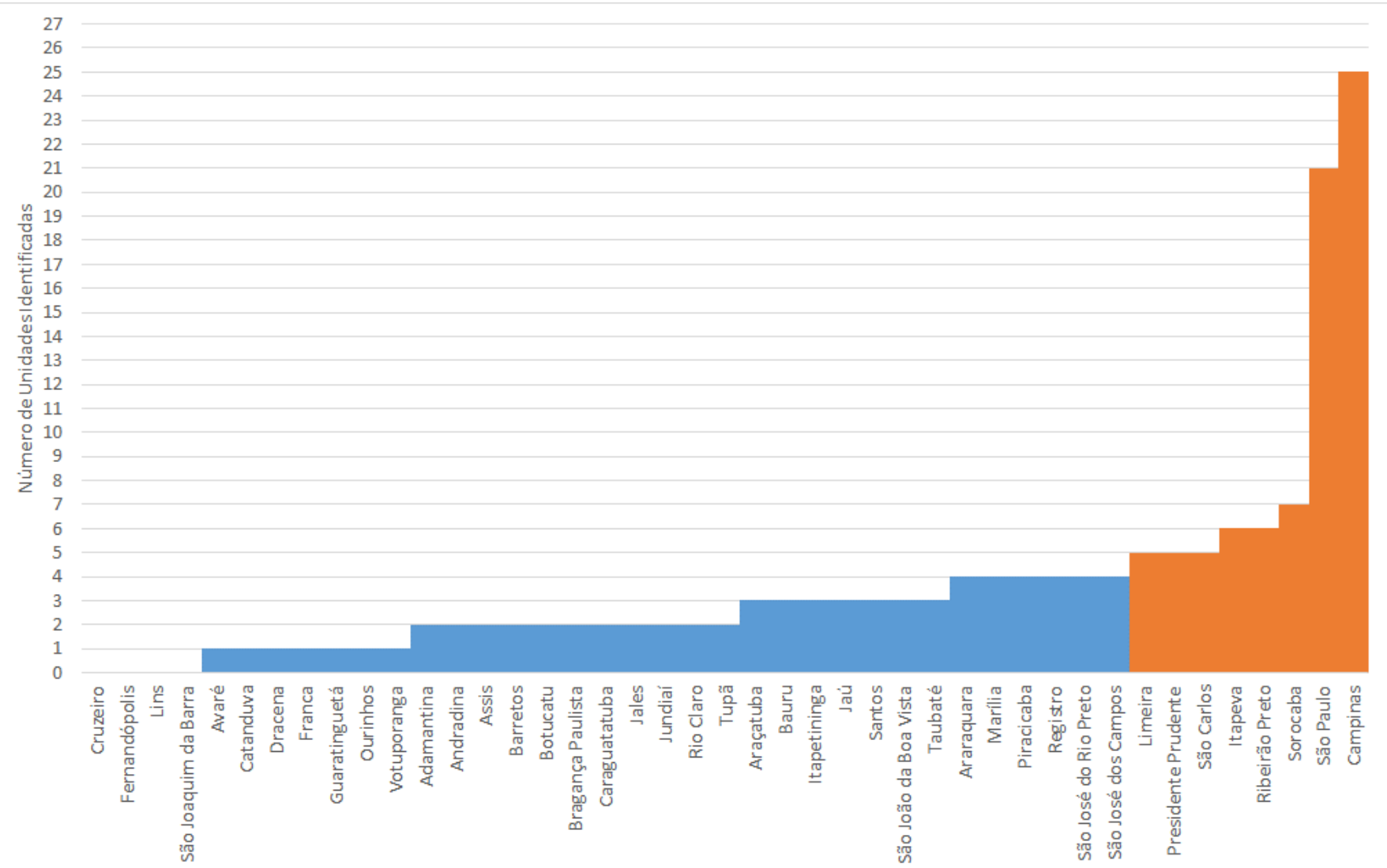

Figura 6. Frequência de distribuição crescente de unidades de ensino superior e/ou pesquisa em Ciências Agrárias.

Fonte: Resultados da Pesquisa.

\section{REFERÊNCIAS BIBLIOGRÁFICAS}

ARAÚJO, V.C. Dimensão local da inovação no Brasil: determinantes e efeitos de proximidade. Tese (Doutorado). São Paulo: Escola Politécnica da Universidade de São Paulo, 189p., 2014.

AUDRETSCH, Bruce. Agglomeration and the location of innovative activity. Oxford review of economic policy, v. 14, n. 2, p. 18-29, 1998.

BRESCHI, S.; MALERBA F. The geography of innovation and economic clustering: some introductory notes. Industrial and Corporate Change, v. 10, n. 4, p. 817-833, dez. 2001.

CEM (Centro de Estudos da Metrópole). Software TerraView Política Social. São Paulo: CEBRAP, 2015. Disponível em: http://www.fflch.usp.br/centrodametropole/1. Acesso em: 20/03/2015.

FIRETTI, Ricardo et al. Similaridades entre municípios do Pontal do Paranapanema: análise de agrupamento em função de características da agropecuária regional. Revista de Economia Agrícola, São Paulo, v. 57, n. 1, p. 19-33, 2010.

GARCIA, R. Economias externas e vantagens competitivas dos produtores em sistemas locais de produção: as visões de Marshall, Krugman e Porter. Ensaios FEE, v. 27, n. 2, p. 301-324, 2006.

GARCIA, R.C.; DA SILVA, C.F.; RIGHI, H.M. Dimensão regional dos esforços de ciência, tecnologia e inovação no Estado de São Paulo. In: BRENTANI, R. R.; BRITO CRUZ, C. H. (Orgs.). Indicadores de ciência, tecnologia e inovação em São Paulo 2010. São Paulo: FAPESP, 2011.

GIL, A.C. Métodos e técnicas de Pesquisa Social. São Paulo: Atlas, 2009.

GONÇALVES, J.S. Dinâmica da agricultura paulista. Informações Econômicas, v.35, n.12, 2005.

GONÇALVES, J.S. Inserção da dimensão da regionalidade na estrutura orçamentária do governo do

Estado de São Paulo. Informações Econômicas, v.36, n.2, p.72-86, 2006. 
MALERBA, F. Sectoral systems of innovation and production. Research policy, v. 31, n. 2, p. 247-264, 2002.

QUADROS, R. et al. Força e fragilidade do sistema de inovação paulista. São Paulo em Perspectiva, v. 14, n. 3, p. 124-141, 2000.

RUFFONI, J.; SUZIGAN, W. Influência da proximidade geográfica na dinâmica inovativa de firmas localizadas em Sistemas Locais de Inovação. Economia, v.13, n. 1, p. 35-66, 2012.

SALLES FILHO, S.. et al. CT\&I no setor agrícola no Estado de São Paulo. In: BRENTANI, R. R.; BRITO CRUZ, C. H. (Orgs.). Indicadores de ciência, tecnologia e inovação em São Paulo 2010. São Paulo: FAPESP, 2011.

SÃO PAULO (Estado). Decreto n. 46.488, de 08 de janeiro de 2002. Reorganiza a Agência Paulista de Tecnologia dos Agronegócios (APTA), da Secretaria de Agricultura e Abastecimento, e dá providências correlatas. Diário Oficial do Estado de São Paulo, 09 jan. 2002.

SEADE [Fundação Sistema Estadual de Análise de Dados]. Regiões administrativas e regiões de governo do estado de São Paulo. Fundação SEADE. 2016. Disponível em: https://www.seade.sp.gov.br. Acesso em: 01/06/2016.

SICSÚ, A.B.; DA SILVEIRA, S.K. Construção de programas de gestão estratégica para Organizações Estaduais de Pesquisa Agropecuária no Brasil: aspectos metodológicos. Navus-Revista de Gestão e Tecnologia, v. 3, n. 1, p. 36-48, 2013.

SUZIGAN, W. et al. . In: Dimensão regional dos esforços de ciência, tecnologia e inovação no Estado de São Paulo. In: Indicadores de ciência, tecnologia e inovação em São Paulo 2004. Coord. LANDI, F. R., São Paulo : FAPESP, 2004.

SUZIGAN, W.; CERRÓN, A. P. M.; DIEGUES JR. A. C. Localização, Inovação e Aglomeração - o papel das instituições de apoio às empresas no Estado de São Paulo. São Paulo em Perspectiva, v.19, no. 2, p.86-100, 2005.

VIEIRA FILHO, J.E.R. Políticas públicas de inovação no setor agropecuário: uma avaliação dos fundos setoriais. Revista Brasileira de Inovação, v. 13, n. 1, p. 109-132, 2013.

VIEIRA, D. Políticas de C\&T e o Desenvolvimento Regional no Estado de São Paulo. 2010. 145 f.

Dissertação de Mestrado - Departamento de Política Científica e Tecnológica, Instituto de Geociências, Universidade Estadual de Campinas, 2010. 